EConomía Teoría y Práctica • Nueva Época, número 47, julio-diciembre 2017, pp. 191-216, http://dx.doi.org/10.24275/ETYPUAM/NE/462017/Sour

\title{
Esfuerzo fiscal de las entidades federativas mexicanas durante el periodo 2004 al 2012*
}

\section{Fiscal efforts of the Mexican states during the period 2004 to 2012}

Delia Laura Sour Vargas**

\section{RESUMEN}

No obstante que la dependencia financiera de los gobiernos municipales hacia el gobierno federal es un problema persistente, poco se ha estudiado el esfuerzo fiscal a nivel estatal en México con evidencia de los últimos años. En este estudio se utilizan datos panel de los 31 estados de la república, excluyendo al Distrito Federal, mediante un modelo de Mínimos Cuadrados Generalizados Factibles (MCGF) para examinar el esfuerzo fiscal estatal. Los resultados extienden las conclusiones de estudios anteriores y se integran al de la literatura sobre el impacto del pacto fiscal sobre las relaciones intergubernamentales en México.

Palabras clave: Descentralización, esfuerzo fiscal, gobiernos locales, México.

Clasificación JEL: H20, H39, H50, H72, H77

\begin{abstract}
Although municipal governments' financial dependency on the federal government is a persistent problem, little attention has been paid to fiscal efforts at the state level in Mexico with data from recent years. This study used panel data from Mexico's 31 states, excluding the Federal District, with a model of Feasible Generalized Least Squares (FGLS), to examine state-level fiscal efforts. The results extend the findings of previous studies and enrich the literature on the impact of recent tax reforms on intergovernmental relations in Mexico.
\end{abstract}

Keywords: Decentralization, Fiscal Effort, Local Governments, Mexico.

JEL Classification: H20, H39, H50, H72, H77

* Fecha de recepción: 14/07/2015. Fecha de aprobación: 20/03/2017

**Universidad Anáhuac México Norte, México. Correo electrónico: laura.sour@anahuac.mx. ORCID 0000-0002-2025-8223 


\section{INTRODUCCIÓN}

Desde 1980 el Sistema Nacional de Coordinación Fiscal (SNCF) regula las relaciones intergubernamentales en los tres niveles de gobierno de México. Bajo este acuerdo las entidades federativas aceptaron ceder la mayor parte de sus potestades tributarias y así el gobierno federal terminó a cargo de la administración de los impuestos de bases amplias (ingreso, IVA e IEPS, principalmente) (Sobarzo, 2003: 3). Por ello, el gobierno federal tiene mayor capacidad de recaudación que los estados, quienes manejan impuestos con bases relativamente reducidas y no todos gravan los mismos impuestos (Sobarzo, 2006: 818). A manera de ejemplo, durante el periodo 20002012, la recaudación total promedio del gobierno federal fue de 9.2 puntos del PIB, mientras que las entidades federativas y los municipios sólo recolectaron el 1.3 puntos (Casares et al., 2015: 534). En respuesta a la trasferencia de casi la totalidad de las responsabilidades fiscales de las entidades federativas, y con el propósito de que los gobiernos locales brinden una mejor atención en la provisión de bienes y servicios públicos, el gobierno federal elaboró un sistema de transferencias intergubernamentales (participaciones). Esta política está sustentada en la teoría que argumenta que los gobiernos subnacionales tienen un mejor conocimiento de los problemas y necesidades que se presentan en su comunidad y, por consiguiente, están mejor informados para destinar los recursos públicos a su cargo y brindar una mejoría en la calidad de vida a sus ciudadanos (Oates, 2003: 1,3).

Adicionalmente, desde la década de los noventa se inició una descentralización del gasto federal hacia los estados y en 1998 se introdujo dentro de los ramos presupuestarios al Ramo 33 y 29. Estos recursos, a diferencia de las participaciones, tienen un destino etiquetado. Desde entonces los estados reciben transferencias intergubernamentales etiquetadas (aportaciones o Ramo 33) y no etiquetadas (participaciones o Ramo 28) (Canavire-Bacarreza et al., 2013: 4). Este esquema de transferencias más que compensa a los gobiernos estatales por la pérdida de sus potestades tributarias sobre el ingreso y el consumo (Sobarzo, 2006: 815).

Sin embargo, el gobierno federal no previó que el diseño del SNCF resultara en que las entidades federativas dejaran de preocuparse por realizar actividades de recaudación y se volvieran dependientes de las transferencias intergubernamentales: en promedio, el 80 por ciento de los ingresos estatales provienen de transferencias y solamente el 20 por ciento de las haciendas estatales se financia con recursos propios, de los cuales el 4.2 por ciento corresponde a ingresos tributarios (Bonet y Rueda, 2011: 1). Es decir, la creación de la coordinación fiscal en México responde a la necesidad de enmendar la concurrencia impositiva y los diversos criterios estatales en materia tributaria, pero, al darse por sentada la autonomía fiscal de los diferentes niveles de gobierno, nunca se pronosticó el crecimiento en 
la dependencia de los gobiernos estatales y municipales hacia las transferencias intergubernamentales (Páramo, 1999: 418). Éste es un gran pendiente de la descentralización fiscal en México: la debilidad en la autonomía financiera de los gobiernos estatales, misma que se agrava con el impacto de la caída de las reservas petroleras a nivel nacional. ${ }^{1}$ De esta manera, desde sus orígenes, el federalismo fiscal en México ha tenido que afrontar la falta de interés de las autoridades locales por generar más recursos, a la par del crecimiento en las necesidades dispares de financiamiento en cada una de las entidades federativas.

La teoría de la descentralización habla de que es importante analizar el balance vertical entre los niveles de gobierno facultados para administrar y decidir la manera en que se ejercen los recursos públicos y su esfuerzo fiscal. El análisis del balance vertical en el caso de México indica que son los estados quienes poseen mayor autonomía y capacidad tributaria en relación con los municipios. Es en este ámbito que se enfoca este trabajo. El objetivo de la presente investigación es analizar el esfuerzo fiscal de las entidades federativas durante el periodo 2004 al 2012, para lo cual se utiliza una base de datos panel de los 31 estados de la república, sin considerar al Distrito Federal. Se pretende probar que las transferencias federales siguen ocasionando desincentivos para el esfuerzo fiscal por parte de las entidades federativas en México mediante el empleo de un modelo de Mínimos Cuadrados Generalizados Factibles (MCGF). Cabe mencionar que una de las principales ventajas de este estimador es su consistencia cuando se aplica a datos tipo panel.

El trabajo consta de seis secciones. La revisión de la literatura sobre la descentralización fiscal se presenta en la primera. En la segunda se describen las diversas metodologías para el cálculo de la capacidad y el esfuerzo fiscal. La revisión de los estudios que examinan con especial énfasis en el caso del esfuerzo fiscal en México -tanto a nivel municipal como estatal- se presenta en la tercera sección. La naturaleza de los datos empleados para el análisis se describe en la cuarta. El modelo a estimar y los resultados obtenidos se presentan en la quinta. La final cierra con las conclusiones del trabajo.

\footnotetext{
${ }^{1}$ Actualmente, el cálculo de las participaciones que reciben los estados se basa en la recaudación federal participable (RFP), la cual se conforma mediante el 100 por ciento de los ingresos generados por el impuesto sobre la renta (ISR), el impuesto al valor agregado (IVA), los impuestos especiales de producción y servicios (IEPS), el impuesto sobre vehículos nuevos (ISAN); y el 85.3 por ciento de los derechos sobre hidrocarburos ingresan al RFP. Este último componente explica la dependencia de las participaciones a la venta de hidrocarburos nacional.
} 


\section{Descentralización FisCAL}

El federalismo fiscal analiza las interrelaciones entre ingreso y gasto de las haciendas públicas en los distintos órdenes de gobierno que conforman a una nación (Oates, 2003: 5). Estas interrelaciones se pueden analizar tanto horizontal como verticalmente. Bajo el primer enfoque se evalúa si los niveles gubernamentales pares poseen la suficiencia para proveer servicios públicos de igual calidad. En el segundo se estudia la correspondencia que existe entre los ingresos y su padrón de responsabilidades de gasto. Aquí es importante mencionar que los ingresos públicos pueden ser propios o provenientes de otro nivel de gobierno. En este último caso, si se analiza a los ingresos transferidos del gobierno federal a los gobiernos locales, se esperaría un balance entre las transferencias intergubernamentales y las responsabilidades de gasto con los ciudadanos. Así entonces el federalismo fiscal es una rama de las finanzas públicas que analiza -tanto de manera positiva como normativa- la forma vertical en la que se entrelazan las haciendas públicas de los diferentes niveles de gobierno.

La descentralización es un concepto intrínseco del federalismo fiscal que consiste en delegar autonomía a los gobiernos locales para el ejercicio de los recursos, con el propósito de obtener mejores metas económicas, políticas y sociales. Con el objetivo en mente de alcanzar la igualdad en oportunidades e ingreso entre las distintas regiones de un país, el gobierno federal otorga transferencias hacia los gobiernos subnacionales (Martinez-Vazquez y Boex, 1997: 5). La premisa subyacente de esta acción es que los gobiernos subnacionales poseen ventaja sobre el gobierno federal debido a que los primeros conocen con mayor claridad las demandas de sus ciudadanos (Martinez-Vazquez y Boex, 1997: 13).

Tres son los niveles en los que la descentralización fiscal se presenta dependiendo del grado de independencia con el que cuentan las autoridades locales para el ejercicio de los recursos públicos (Bird y Villancourt, 1998: 3). En la desconcentración sólo existe una dispersión de responsabilidades del gobierno federal hacia los gobiernos subnacionales. La delegación se presenta cuando el gobierno local actúa como agente del gobierno central ejecutando ciertas funciones encomendadas, mientras que en la devolución el gobierno local recibe la autoridad para decidir e implementar sus propias políticas. Entonces una descentralización administrativa se presenta cuando se distribuyen recursos a los municipios bajo la directriz del gobierno federal (Tanzi, 1996: 297). En el caso de que los gobiernos subnacionales sean facultados para administrar y decidir la manera en que ejercen los recursos se considera que existe una descentralización fiscal.

Uno de los principales argumentos económicos a favor de la descentralización fiscal (Martinez-Vazquez y McNab (2003: 1600) destaca la importancia de analizar 
la eficiencia de los gobiernos subnacionales respecto al gobierno federal, ya que los primeros debieran conocer mejor las demandas de sus ciudadanos. Lo anterior coincide con el marco normativo de la literatura acerca del federalismo fiscal donde el objetivo es el fortalecimiento económico de la nación y el abastecimiento eficaz de los servicios públicos. Así, dentro del contexto de la descentralización tributaria, Aguilar y Andino (2009: 26) consideran relevante la medición del esfuerzo fiscal, debido a que éste se puede usar como una aproximación parcial de la eficiencia en la recaudación y la utilización de la capacidad recaudatoria para generar ingresos propios por parte de los gobiernos subnacionales.

\section{LOS CONCEPTOS DE CAPACIDAD Y ESFUERZO FISCAL}

Si bien una pieza importante en la descentralización fiscal es la autonomía para el ejercicio de los recursos, otro aspecto no menos importante es la capacidad de tributación con la que cuentan los diversos niveles de gobierno. En términos generales, la capacidad fiscal es el máximo ingreso tributario que se puede obtener considerando las características económicas, sociales, institucionales y demográficas en una determinada región (Pessino y Fenochietto, 2010: 66). A pesar de que existe un consenso a nivel conceptual sobre lo que se entiende por capacidad fiscal, el debate continúa sobre cuál es la mejor forma de estimar esta cifra, pues la potencialidad de una región depende tanto de las tasas impositivas como del grado y naturaleza de la actividad económica. Para instrumentar la medición de la capacidad fiscal existen varios métodos. ${ }^{2}$ Por ejemplo, el Sistema Fiscal Representativo (SFR) estima la capacidad fiscal suponiendo que una entidad federativa realizará su esfuerzo tributario promedio. Para obtener este cálculo se requiere información sobre las tasas impositivas efectivas en cada estado y para cada impuesto en particular. Estos datos se aplican a una base gravable que razonablemente sea un buen proxy de la capacidad fiscal de la región. Así, y una vez que se divide a la recaudación real entre la potencial, se construye un índice del uso de la potencialidad fiscal (IUPF) y se procede a ordenar a las entidades federativas con base en su esfuerzo fiscal (Sobarzo, 2006: 823). ${ }^{3}$ Es decir, ante la ausencia de una medida absoluta de la potencialidad fiscal, se puede comparar a cada estado con el promedio

\footnotetext{
${ }^{2}$ Martinez-Vazquez y Boex (1997: 18) explica cinco métodos diferentes para instrumentar la medición de la capacidad fiscal, con sus ventajas y desventajas.

${ }^{3} \mathrm{El}$ esfuerzo fiscal se puede representar matemáticamente como: esfuerzo fiscal = recaudación actual/ capacidad fiscal (Sour, 2008: 273). Así, y con base en la estimación de la capacidad fiscal que se elija, se pueden construir estimaciones del esfuerzo fiscal más robustas ya sea en términos de desagregación de componente o en la actualización de datos.
} 
nacional para un impuesto en particular, un grupo de impuestos o todo el sistema fiscal. La ventaja principal de este método es que permite observar la recaudación de manera desagregada por impuesto y estado, lo que lo convierte en el método más deseado para estimar el ingreso tributario máximo. Desafortunadamente este tipo de estimación demanda altos contenidos de información sobre el origen territorial de los impuestos en el país, por lo que a la fecha se han hecho pocos trabajos para México, entre los que destacan los documentos de Sobarzo (2006) y Bonet y Rueda (2011).

El cálculo del SFR escapa de los objetivos de este trabajo debido a que requiere una gran cantidad de información para todas las entidades federativas y que además ésta sea consistente entre sí. Esto explica el por qué los análisis que han empleado esta técnica utilizan cifras con más de 10 años de antigüedad (2007). La falta de información desagregada y confiable es un problema grave al que se enfrenta el investigador para realizar este tipo de análisis (Martinez-Vázquez y Boex, 1997: 18). Así el estudio de las capacidades fiscales se torna en un problema empírico. El presente trabajo tiene como objetivo presentar un análisis de actualidad, por lo que se sacrifica desagregación al estimar la capacidad fiscal utilizando el PIB estatal. De esta manera se considera al valor total de los bienes y servicios producidos en la entidad durante el periodo de un año. De acuerdo con las cuentas nacionales, el valor de la producción es igual al pago de todos los factores productivos (tierra, trabajo y capital), porque refleja el ingreso que recibieron las empresas y los trabajadores en un determinado periodo de tiempo. En este sentido, el PIB es una buena aproximación al total de los recursos potencialmente gravables. Es aquí donde toma relevancia el estudio y la medición del esfuerzo fiscal para los estudiosos de la descentralización fiscal.

\section{Esfuerzo FISCAL EN MÉXICO}

Es evidente que la estimación de la capacidad fiscal, elemento necesario para calcular el esfuerzo tributario, requiere de una amplia disponibilidad de datos para el nivel de gobierno que se quiera analizar. Así las cosas, el reto se magnifica si lo que se busca es comparar diversos países, donde la disponibilidad y la desagregación con la que se generan los datos en cada región puede llegar a ser muy heterogénea. Por ello, la revisión de estos estudios internacionales debe ser muy cuidadosa. ${ }^{4}$ En aras de buscar la mayor homogeneidad posible y la congruencia en las

\footnotetext{
${ }^{4}$ A manera de ejemplo véase el trabajo de Pessino y Fenochietto (2010), quienes estudian los determinantes de 96 países, y de Ramírez y Bedoya (2014), quienes analizan el caso colombiano.
} 
cifras de las cuentas nacionales de los diferentes niveles de gobierno, a continuación se presentará el detalle de la literatura sobre el caso de México.

\section{Estudios a nivel municipal}

Varios estudios han analizado la relación entre el diseño del acuerdo fiscal intergubernamental mexicano y el esfuerzo tributario de los gobiernos subnacionales. ${ }^{5}$ La principal conclusión de estos estudios es que el modelo de coordinación vigente propicia la dependencia en los diferentes niveles de gobierno. Uno de los primeros investigadores en emplear cifras agregadas para realizar un análisis estadístico es Raich (2003: 1-2,31), quien analiza una muestra de 217 municipios del estado de Puebla durante el periodo 1993-2000, para capturar el antes y el después de la creación del Fondo de Aportaciones para la Infraestructura Social Municipal (FAISM) perteneciente al Ramo 33. Su objetivo es estudiar si el aumento en las transferencias federales generó un cambio en el esfuerzo fiscal de los municipios en lo relacionado con el impuesto predial y los derechos de agua. El autor encuentra un impacto negativo sobre el esfuerzo fiscal en los municipios estudiados en Puebla.

Moreno (2003: 1, 9, 14-15) examinó los efectos de las transferencias federales sobre el logaritmo de los ingresos tributarios per cápita utilizando tres diferentes modelos econométricos para los años 1998 y 1999. Las tres especificaciones señalan que la creación del Ramo 33 en conjunto con la existencia de las transferencias no condicionadas (Ramo 28) muestran una relación negativa y significativa con los ingresos tributarios municipales.

Ibarra, Sandoval y Sotres (2001) estudian los ingresos de los municipios mexicanos para el periodo 1975-1995 mediante un análisis de diferencias de medias. Encuentran que tanto el Sistema de Coordinación Fiscal como la reforma al artículo 115 constitucional han causado que las haciendas públicas de los municipios sean más dependientes de las participaciones.

Con una base de datos panel de 2,412 municipios, durante el periodo de 1993 a 2004, Sour (2008: 272-274, 282) encontró que los dos tipos de transferencias intergubernamentales han ocasionado una relación negativa con el esfuerzo fiscal de los gobiernos locales de México. También presenta evidencia que cuestiona el que los municipios grandes realicen un mayor esfuerzo fiscal en comparación con los pequeños.

\footnotetext{
${ }^{5}$ Para mayor detalle de los primeros estudios realizados, véase Sempere y Sobarzo (1994), Arellano (1996) y Courchene, Díaz Cayeros y Webb (2000).
} 
Ibarra-Salazar y Mollick (2011: 1, 9, 20, 22-23) evaluaron el efecto de las transferencias federales en el esfuerzo fiscal de 2,287 municipios mexicanos y 3,083 condados estadunidenses situados en la frontera México-Estados Unidos para el año 2000. La hipótesis a probar es que el efecto de las transferencias intergubernamentales sobre el esfuerzo fiscal es diferente para los municipios situados en la frontera, en comparación con los no fronterizos. Los autores encontraron que las transferencias ocasionan un efecto negativo sólo en los municipios no fronterizos, a diferencia del caso de Estados Unidos donde las transferencias, federales y estatales en los condados no fronterizos fue positivo, y negativo para los fronterizos.

Canavire-Bacarreza y Zuñiga (2013: 17, 20,23) investigan el impacto de las transferencias federales sobre el esfuerzo fiscal de los municipios del estado de Sinaloa para el periodo de 1993 a 2008 utilizando dos especificaciones de la variable dependiente. Los resultados obtenidos muestran que el sistema de transferencias genera impactos negativos en las haciendas públicas de los gobiernos subnacionales debido a que otorga incentivos para la disminución del esfuerzo fiscal.

\section{Estudios a nivel estatal}

Sobarzo $(2003: 8,18,21)$ estima las capacidades y el potencial tributario de los estados en México usando como parámetro el desempeño potencial tributario del país. Primero calculó la tasa efectiva de los distintos impuestos estatales y posteriormente la comparó con la tasa efectiva promedio no ponderada de todos los estados. Al multiplicar esta tasa por la base tributaria, obtuvo el potencial tributario en términos absolutos de cada entidad federativa, la cual representa el monto del ingreso tributario que cada estado podría recaudar, si se gravara el promedio de la base potencial. El autor, al ajustar los datos basándose en la población de cada estado y estimando la brecha per cápita, descubrió que el comportamiento tributario de los gobiernos subnacionales está condicionado por su grado de desarrollo económico.

Un primer intento por estudiar la capacidad administrativa, fiscal, financiera y la provisión de servicios de los gobiernos estatales de manera global lo realizan Ibarra-Salazar y Sotres (2005: 190). Con este objetivo en mente desarrollan un índice de desempeño para las entidades federativas utilizando cifras de 1997. Debido a que obtienen resultados consistentes mediante tres estimaciones econométricas (mínimos cuadrados ordinarios, ordered probit y ordered logit), los autores concluyen que las mejorías en el desempeño global de los estados están positivamente relacionadas con el ingreso per cápita de los habitantes, la cercanía 
con la frontera norte del país, y negativamente con el índice de marginación y el tamaño de la población.

Sobarzo (2006: 844) construye un Sistema Fiscal Representativo (SFR) y encuentra que el desempeño de las entidades federativas se determina principalmente por el número de impuestos que se recauda en las entidades. Asimismo, también concluye que los estados con mejores y peores desempeños son aquellos que tienen capitales grandes. Entonces parece que las metrópolis concentran la mayor actividad económica, pero también los problemas de informalidad y la evasión, lo que repercute en el desempeño de sus funciones fiscales.

Bonet y Rueda (2011: 15) calculan dos indicadores de esfuerzo fiscal estatal: el Índice de Esfuerzo Fiscal (IEF) y el Índice de Uso Potencial Fiscal (IUPF) para los estados del país durante los años del 2003 al 2007. El IEF aproxima el esfuerzo global fiscal de cada estado y el IUPF evalúa a cada entidad en lo relacionado con el impuesto a la nómina. El propósito de estos autores es encontrar cuáles son las variables que explican las diferencias en el esfuerzo observado entre diversas entidades. Encuentran que el mayor nivel de ingreso per cápita junto con el grado de informalidad en el mercado laboral explican un porcentaje importante de las desigualdades observadas. Argumentan que ya se está explotando un alto porcentaje del impuesto a la nómina por lo que sugieren que para aumentar la recaudación se disminuya la evasión y la elusión fiscal en las entidades federativas.

Es conveniente resaltar que la cantidad de estudios sobre el esfuerzo fiscal a nivel estatal para el caso de México no es tan amplia como la de los análisis municipales, a pesar de que los primeros poseen más autonomía fiscal que los municipios. Entonces, de conformidad con el marco de atribuciones del SNCF, las entidades federativas deberían ser las unidades de estudio en los trabajos sobre esfuerzo fiscal debido a que cuentan con mayor autonomía y capacidad de recolección tributaria. En la siguiente sección se analizan a las entidades federativas en México durante el periodo de años más largo que se tiene conocimiento (11 años) y se realizan varias pruebas para verificar la robustez de los coeficientes estimados en relación con el esfuerzo tributario observado en ese periodo de tiempo.

\section{DATOS}

El objetivo de este trabajo es mostrar a nivel estatal el efecto que las transferencias intergubernamentales condicionadas y no condicionadas han tenido sobre el esfuerzo fiscal de las entidades federativas del 2004 al 2012, periodo no analizado en la literatura. Se analizan las finanzas públicas y el esfuerzo fiscal de los 31 estados de la república, excluyendo del estudio al Distrito Federal, debido a que este último presenta una naturaleza fiscal y jurídica diferente a la de las demás entidades. 
Para la construcción de la base de datos se utilizaron dos fuentes de información: la primera fue el Instituto Nacional de Estadística y Geografía (INEGI) de donde provienen las transferencias federales (etiquetadas y no etiquetadas), el Indicador Trimestral de la Actividad Económica Estatal (ITAEE) base 2008 y el PIB a precios corrientes. La segunda fuente de información es el Sistema Estatal y Municipal de Bases de Datos (SIMBAD) ${ }^{6}$ de donde se obtuvieron los ingresos estatales (propios y totales).

Como se mencionó, existen diferentes formas para estimar el esfuerzo fiscal. En este trabajo se define como la proporción de los ingresos propios estatales ${ }^{7}$ entre el PIB estatal. ${ }^{8}$ Las variables independientes que se utilizan son: las participaciones federales $(P)$ del Ramo 28 entre los ingresos totales estatales, las aportaciones federales $(A)$ del Ramo 33 entre los ingresos totales estatales y el Índice Trimestral de Actividad Económica Estatal (IT).

La proporción de las participaciones federales entre los ingresos totales estatales se incluyó como variable independiente para poder evaluar los cambios en el esfuerzo fiscal de los gobiernos subnacionales, debido a que las entidades gozan de libre albedrío para decidir cómo emplear estos recursos. Se busca probar que al existir un ingreso proveniente del gobierno federal que no es etiquetado, los estados tienen incentivos para disminuir su esfuerzo por captar ingresos propios, generando una mayor dependencia del gobierno central. Por tales motivos, el signo esperado de esta variable es negativo.

La proporción de las aportaciones federales respecto a los ingresos totales de las entidades federativas sirve para valorar el efecto que dichas transferencias etiquetadas ocasionan en la recaudación de los ingresos propios por parte de las entidades federativas. Al existir un destino preestablecido por parte del gobierno central sobre la forma de administrar y ejercer tales recursos, existe cierta posibilidad de que las entidades federativas disminuyan su propia recaudación. Se prevé que el efecto de esta variable independiente sobre el esfuerzo fiscal también sea negativo.

La tercera variable independiente es el Índice Trimestral de Actividad Económica Estatal (ITAEE), un indicador de coyuntura de la situación macroeconómica de las entidades federativas, el cual se compone con la información de las actividades

\footnotetext{
${ }^{6}$ Aplicación del INEGI que brinda diversos conjuntos de estadísticas presentadas en formato desagregado por estados o municipios.

${ }^{7}$ Conformados por los ingresos tributarios, el cobro de derechos y aprovechamientos y productos, así como las contribuciones de mejoras.

${ }^{8}$ Se emplea el PIB con el objetivo de poder cuantificar la producción estatal expresada en términos monetarios (millones de pesos a precios corrientes, teniendo como año base el 2008).
} 
económicas primarias, secundarias y terciarias. Debido a que el esfuerzo físcal se construye como la razón de los ingresos propios estatales entre el PIB estatal, se puede emplear el ITAEE como variable, puesto que no hay evidencia de que se pueda presentar algún problema de autocorrelación con el esfuerzo fiscal. ${ }^{9}$ Se espera que el efecto del ITAEE en el esfuerzo fiscal sea positivo, debido a que un aumento en la producción y la actividad empresarial se puede traducir en mayores ingresos propios y por ende mayores recursos para las arcas estatales.

\section{ESPECIFICACIÓN DEL MODELO Y RESULTADOS}

La estimación del esfuerzo fiscal se realiza empleando las variables expresadas en logaritmos naturales para eliminar factores de escala e interpretar los coeficientes estimados como elasticidades..$^{10} \mathrm{La}$ forma funcional es la siguiente manera:

$$
\log I_{i t}=\alpha_{0}+\beta_{1} \log P_{i t}+\beta_{2} \log A_{i t}+\beta_{3} \log I T_{i t}+e_{i t}
$$

\section{Donde:}

$\alpha=$ Constante

$\beta_{1}=$ Coeficiente de Participaciones

$\beta_{2}=$ Coeficiente de Aportaciones

$\beta_{3}=$ Coeficiente del Índice de Actividad Económica Estatal

$I_{i t}=$ Ingresos propios estatales entre el PIB estatal en la entidad federativa i para el año $t$

$P_{i t}=$ Participaciones entre los ingresos totales estatales

$A_{i t}=$ Aportaciones entre los ingresos totales estatales

$I T_{i t}=$ Índice de Actividad Económica Estatal

$\mathrm{e}=$ Término de error

$i=$ Entidad federativa $(i=1 \ldots 31)$

$$
\mathrm{t}=\text { Año }(t=1 \ldots 9)
$$

\footnotetext{
${ }^{9}$ Para construir esta variable, se promediaron los cuatro trimestres, ya que no se publica el valor anual consolidado.

${ }^{10}$ Los logaritmos permiten dar un tratamiento a la información numérica, donde se controla la disparidad que existe entre las diversas series, manteniendo la proporcionalidad entre las mismas.
} 
Tanto $\beta_{I,} \beta_{2}$ y $\beta_{3}$ son constantes, donde se espera que $\beta_{3}$ sea positivo y los demás coeficientes negativos. Primero se estimaron los modelos pooled, de efectos fijos y de efectos aleatorios (Anexos 1, 2 y 3). Para seleccionar cuál estimación es la mejor se realizó la prueba de Hausman (Anexo 4). Se determina que el mejor modelo es el de efectos aleatorios. ${ }^{11}$ Se prueba autocorrelación con la prueba de Wooldridge (Anexo 5). ${ }^{12}$ Para poder comprobar si existe heterocedasticidad en el modelo estimado se empleó la prueba del Multiplicador Lagrangiano de Breusch y Pagan, de efectos aleatorios, con la cual se determinó que el modelo presenta problemas de heterocedasticidad. ${ }^{13}$ Por consiguiente, el modelo empleado no es eficiente, es decir, el estimador no posee mínima varianza. Por tal motivo, se estima el método de Mínimos Cuadrados Generalizados Factibles (MCGF). ${ }^{14}$ Posteriormente, se verifica la no presencia de variables omitidas en el modelo mediante la prueba de Ramsey Reset. ${ }^{15}$ Con dicha prueba se confirma la ausencia de variable omitida (Anexo 8). Los resultados obtenidos con el MCGF se presentan a continuación:

\footnotetext{
${ }^{11}$ Utilizamos la prueba de Hausman para elegir entre el modelo de efectos fijos y el de efectos aleatorios. La hipótesis nula de la prueba de Hausman indica que el modelo de efectos aleatorios es el estimador más eficiente. La hipótesis alternativa es que las estimaciones del modelo de efectos aleatorios son sesgadas e inconsistentes. En otras palabras, al rechazar la hipótesis nula tenemos suficiente evidencia para aceptar que el modelo de efectos fijos es consistente.

${ }^{12}$ En aras de asegurar que el modelo no presentara algún problema de multicolinealidad, aunque es poco común usando datos en panel, se realizó la prueba de los Factores de Inflación de Varianza (VIF). Esta prueba se utiliza para estimar la severidad de la multicolinealidad en la regresión. En la prueba se observó que el modelo no presentó problemas de colinealidad, ya que los valores obtenidos no son mayores que 10 .

${ }^{13}$ Esta prueba compara los efectos de las condiciones de máxima verosimilitud para detectar si la varianza de los residuales es igual a cero. Con este objetivo se emplea un multiplicador lagrangeano. Esta prueba aparece en el Anexo 6.

${ }^{14}$ En el Anexo 7 aparecen los resultados de esta estimación. El MCGF se obtiene a partir del modelo lineal transformando las variables por una matriz simétrica invertible. Es decir, con este método se estima la matriz de varianza-covarianza y posteriormente se calcula el estimador MCGF, sustituyendo el valor de dicha matriz por su estimador. Las propiedades de este estimador de la varianza son las mismas propiedades que poseen los estimadores lineales: estimadores consistentes, asintóticamente normales, insesgados y eficientes.

${ }^{15}$ La prueba consiste en correr la regresión original, se guarda la variable dependiente estimada y posteriormente se eleva al cuadrado. Luego se vuelve a correr la regresión original, pero se le agrega la variable dependiente estimada al cuadrado como variable independiente. En caso de que saliera significativa la variable dependiente estimada al cuadrado, se comprobaría la existencia de una variable omitida (Del Carpio, 2008: 28).
} 
Tabla 1. Resultados

\begin{tabular}{|c|c|}
\hline VARIABLE INDEPENDIENTE & \\
\hline \multirow{2}{*}{ Participaciones } & $-0.26 * *$ \\
\hline & {$[0.081]$} \\
\hline \multirow{2}{*}{ Aportaciones } & $-0.176^{* * *}$ \\
\hline & [0.078] \\
\hline \multirow{2}{*}{ ITAEE } & $0.797 * * *$ \\
\hline & {$[0.217]$} \\
\hline \multirow{2}{*}{ Constante } & $-9.055^{* * *}$ \\
\hline & [0.953] \\
\hline Observaciones & 279 \\
\hline Wald chi-squared & 43.21 \\
\hline \multicolumn{2}{|c|}{ * significante al $10 \%$; ** significante al $5 \%$; *** significante al $1 \%$} \\
\hline \multicolumn{2}{|c|}{ Errores estándar entre corchetes } \\
\hline
\end{tabular}

Los resultados indican que existe una relación negativa y estadísticamente significativa entre las transferencias federales y el esfuerzo fiscal de las entidades federativas. Es decir, los gobiernos subnacionales, al recibir transferencias no etiquetadas (Ramo 28) presentaron una disminución de sus ingresos propios. De igual manera, el recibir transferencias etiquetadas por parte del gobierno federal ha ocasionado un impacto negativo en el esfuerzo de las entidades federativas por conseguir ingresos por sus propios medios. Sin embargo, el parámetro asociado a las participaciones es más grande que el de las aportaciones. Esta diferencia muestra que los recursos etiquetados tienen una menor relación con el esfuerzo fiscal que la que presentan las aportaciones. Este resultado contribuye a la discusión sobre el diseño de reformas fiscales que aspiran a cerrar las brechas sociales en las zonas más marginadas, y a la vez incentivar la recaudación de recursos propios. ${ }^{16}$ Finalmente, la actividad económica medida a través del ITAEE presenta una relación positiva y estadísticamente significativa con el esfuerzo fiscal de los estados. Es decir, ante una mayor actividad industrial y económica, las entidades federativas tienen posibilidades de que su esfuerzo fiscal aumente.

Estos resultados complementan la evidencia presentada a nivel municipal. Los datos ratifican que el acuerdo fiscal intergubernamental vigente en el país ha derivado en una dependencia de los gobiernos estatales por recursos interguber-

\footnotetext{
${ }^{16}$ En este sentido véase el trabajo de Casares et al. (2015) para el caso específico de México.
} 
namentales. En este sentido se complementa la literatura existente sobre el tema de descentralización fiscal en México.

\section{Conclusiones}

El federalismo de la hacienda pública en México inició en 1954 a partir de la promulgación de la Ley de Coordinación Fiscal (LCF), la cual sentó las bases de la normatividad sobre la distribución de los recursos públicos del país. En 1980 los gobiernos estatales aceptaron ceder al gobierno federal sus potestades tributarias sobre el ingreso y el consumo, a cambio de recibir transferencias federales no etiquetadas o no condicionadas en forma de participaciones mediante el Ramo 28.

Posteriormente, en 1998, a partir de las modificaciones a la LCF, se creó el Ramo 33 para transferir las aportaciones federales a los gobiernos subnacionales, con el objetivo de fortalecer los recursos estatales destinados a los rubros que se encaminan al crecimiento y desarrollo social. El Ramo 33 también se le conoce como transferencias intergubernamentales condicionadas y se constituye por varios fondos enfocados en los rubros tales como educación, salud, infraestructura, seguridad pública y desarrollo social. La principal diferencia entre las transferencias federales condicionadas y no condicionadas radica en la libertad con la que los gobiernos subnacionales ejercen estos últimos. De esta forma, en la actualidad los ingresos de las entidades federativas constan de dos rubros principalmente. En el primero están las transferencias intergubernamentales (integradas por el Ramo 28 y el Ramo 33), y en el segundo están los ingresos propios.

En este trabajo se comprueba la baja capacidad de las entidades federativas para dar holgura financiera a las arcas de los gobiernos y realizar así sus funciones operativas. Es decir, para el periodo 2004 a 2012 los estados básicamente sólo han contado con las transferencias intergubernamentales para invertir en obras públicas, programas sociales, y de manera general, dar un mejor cumplimiento a las demandas ciudadanas. A la luz de la revisión de estudios estatales sobre el esfuerzo fiscal se puede decir que sigue dependiendo del nivel de desarrollo económico y el crecimiento del país como alternativa para mejorar el desempeño fiscal.

Los resultados de esta investigación ratifican las conclusiones de estudios anteriores, pero también extienden la discusión sobre el impacto del acuerdo fiscal sobre las relaciones intergubernamentales en el país. Cada día resulta más apremiante desarrollar una línea de investigación sobre la factibilidad política y económica de una descentralización fiscal profunda en México. Se debe sumar a esta discusión a todos los agentes de la política en los diferentes niveles gubernamentales para repensar las leyes de hacienda, fiscales y de coordinación fiscal de la república y de las entidades federativas. En este ejercicio un eje rector del debate 
debe ser la partición de competencias fiscales que contribuya a un mayor esfuerzo fiscal, así como también a mejorar la asignación del erario público. Lo anterior sin olvidar las restricciones que la informalidad y la elusión imponen a las bases tributarias. Estos hechos toman mayor relevancia sobre todo a la luz de la disminución de las reservas petroleras, principal fuente de financiamiento de las finanzas públicas mexicanas, y por ende de los ingresos estatales en el país. Siempre hay que tener presente que una de las aspiraciones del federalismo es la convivencia de distintas soberanías con el objetivo de alcanzar la unidad nacional mediante la integración de los gobiernos subnacionales. Su éxito radica en la capacidad de lograr la conjunción armónica entre el gobierno federal y la autonomía de los estados.

\section{REFERENCIAS BIBLIOGRÁFICAS}

Aguilar, Víctor y Andino, Mauro (2009), "Esfuerzo fiscal a nivel nacional y provincial, Ecuador 2001-2007", Notas Tributarias, núm. 2009-03, Departamento de Estudios Tributarios.

Arellano, Rogelio (1996), "Necesidades de cambio en las relaciones hacendarias intergubernamentales en México", en Alicia Hernández (coord.), ¿Hacia un nuevo federalismo, México, Fondo de Cultura Económica, pp. 109-152.

Bird, Richard y Villancourt, Francois (1998), "Fiscal decentralization in developing countries: an overview", Fiscal Decentralization in Developing Countries, Cambridge University Press.

Bonet, Jaime y Rueda, Fabio (2011), "Esfuerzo fiscal en los estados mexicanos", Documento elaborado para el XXIII Seminario Regional de Política Fiscal, Chile, Banco Interamericano de Desarrollo.

Canavire-Bacarreza, Gustavo y Zuñiga, Nicolás (2013), "Fiscal transfers a curse or blessing? Examining their effect on local taxation in Mexico", International Center for Public Policy, Andrew Young School of Policy Studies.

Casares, Enrique; García, María Guadalupe; Ruiz, Lucía y Sobarzo, Horacio (2015), "Income Distribution, Taxes and Tranfers in Mexico An applied analysis of general equilibrium", El Trimestre Económico, 82 (327), pp. 523-558.

Courchene, Thomas; Díaz Cayeros, Alberto y Webb, Steven (2000), "Transfers and the Nature of the Mexican Federation", en Marcelo M. Giugale, y Steven Webb (eds.), Achievevements and Challenges of Fiscal Decentralization. Lessons from Mexico, Washington, World Bank, pp. 200-236. 
Del Carpio, Jorge Edwig (2008), "Manual de staTA 10.0.", DC CONSULTING, disponible en http://ciede.net/econometrias/manual_stata10_jdc.pdf

Ibarra Salazar, Jorge; Sandoval Musi, Alfredo, y Sotres Cervantes, Lida (2001), "Participaciones federales y dependencia de los gobiernos municipales en México, 1975-1995", Investigación Económica, (237), pp. 25-62. (2005), "Variables que explican el desempeño de los gobiernos estatales mexicanos", Gestión y Política Pública, 14 (1), pp. 169-196.

Ibarra Salazar, Jorge y Mollick, André Varella (2011), "Fiscal Effort and the Borderlands: Evidence of the US-Mexico Border", The Puentes Consortium 's 2nd Annual Symposium on Us-México Border Security Border, Security and Economic Development at the US-Mexico Border.

INEGI (2013), "Indicador Trimestral de Actividad Económica Estatal", Fuentes y Metodologías, Sistema de Cuentas Nacionales de México.

Martinez-Vazquez, Jorge y Boex, Jameson (1997), "Fiscal Capacity: An overview of concepts and measurement issues and their applicability in the Russian Federation", Andrew Young School of Policy Studies Working Paper, (973), Georgia State University.

Martinez-Vazquez, Jorge, y McNab, Robert (2003), "Fiscal decentralization and economic growth", World Development, 31 (9), pp. 1597-1616.

Moreno, Carlos L. (2003), "Fiscal Performance of Local Governments in México; The Role of Federal Transfers", Centro de Investigación y Docencia Económicas, Documento de Trabajo 127.

Oates, Wallace E. (2003), "An Essay on Fiscal Federalism", Economic Perspective, 11 (1), pp. 169-78.

Páramo Colmenares, David (1999), "Retos del federalismo fiscal mexicano", Comercio Exterior, 49 (5), pp. 415-431.

Pessino, Carola y Fenochietto, Ricardo (2010), "Determining countries' tax effort", Hacienda Pública Española, 195 (4), pp. 65-87.

Ramírez, Juan Mauricio y Bedoya, Juan Guillermo (2014), "Regalías directas por hidrocarburos y esfuerzo fiscal municipal en Colombia", Coyuntura Económica, 44 (2), pp. 15-34.

Raich, Uri (2003), "Impacts of expenditure decentralization on Mexican local governments", Working Paper of the Department of Public Administration, núm. 102, Centro de Investigación y Docencia Económicas.

Sempere, Jaime y Sobarzo, Horacio (1994), La descentralización fiscal en México: algunas propuestas, Centro de Estudios Económicos, El Colegio de México. 
Sobarzo, Horacio (2003), "México: estudio sobre la capacidad fiscal y tributaria y el esfuerzo tributario de los gobiernos estatales en México", Documento elaborado para el Foro Enfoques Alternativos de Federalismo Fiscal en México, México, Indetec. (2006), "Esfuerzo y potencialidad fiscal de los gobiernos estatales en México: un sistema fiscal representativo", El Trimestre Económico, vol. LXXIII, núm. 292, pp. 809-861.

Sour, Laura (2008), "Un repaso de conceptos sobre capacidad y esfuerzo fiscal, y su aplicación para los gobiernos locales mexicanos", Estudios Demográficos y Urbanos, 23 (2), pp. 271-297.

Tanzi, Vito (1996), "Fiscal federalism and decentralization: a review of some efficiency and macroeconomic aspects", In Annual World Bank conference on development economics, pp. 295-317. 
208 ECONOMÍA TEORÍA Y PRÁCTICA • Nueva Época, número 47, julio-diciembre 2017

\section{Anexo 1. Modelo Pooled}

\begin{tabular}{|r|l|}
\hline .reg In_esfuerzo_fisca In_part In_aport In_itaee \\
\hline Number of obs $=$ & 9 \\
\hline $\mathrm{F}(3,275)=$ & 0.700 \\
\hline Prob $>\mathrm{F}=$ & 0.553 \\
\hline R-squared $=$ & 0.008 \\
\hline Adj R-squared $=$ & -0.003 \\
\hline
\end{tabular}

\begin{tabular}{|c|c|c|c|c|}
\hline & Coef. & Std. Err. & $\mathrm{t}$ & P>|t| \\
\hline In_part & 0.018 & 0.016 & 1.157 & 0.248 \\
\hline In_aport & 0.013 & 0.011 & 1.181 & 0.239 \\
\hline In_itaee & 0.000 & 0.000 & 0.771 & 0.442 \\
\hline _cons & -0.010 & 0.016 & -0.618 & 0.537 \\
\hline
\end{tabular}




\section{Anexo 2. Efectos fijos}

\begin{tabular}{|c|c|c|c|}
\hline \multicolumn{4}{|c|}{.xtreg In_esf_fis In_part In_aport In_itaee, fe } \\
\hline \multicolumn{2}{|c|}{ Fixed-effects (within) regression } & Number of obs $=$ & 279 \\
\hline \multicolumn{2}{|l|}{ Group variable: entidad } & Number of groups $=$ & 31 \\
\hline R-sq: within = & 0.225 & Obs per group: $\min =$ & 9 \\
\hline between $=$ & 0.024 & $\operatorname{avg}=$ & 9 \\
\hline \multirow[t]{2}{*}{ overall = } & 0.000 & $\max =$ & 9 \\
\hline & & $F(3,245)=$ & 23.68 \\
\hline $\operatorname{corr}\left(u_{-} \mathrm{i}_{1}, \mathrm{Xb}\right)=$ & -0.284 & Prob $>F=$ & 0.000 \\
\hline
\end{tabular}

\begin{tabular}{|r|l|}
\hline Number of obs $=$ & 279 \\
\hline Number of groups $=$ & 31 \\
\hline & \\
\hline Obs per group: $\min =$ & 9 \\
\hline $\mathrm{avg}=$ & 9 \\
\hline $\max =$ & 9 \\
\hline & \\
\hline $\mathrm{F}(3,245)=$ & 23.68 \\
\hline Prob $>\mathrm{F}=$ & 0.000 \\
\hline
\end{tabular}

\begin{tabular}{|l|c|c|c|c|c|c|}
\hline in_esf_fia & Coef. & \multicolumn{1}{|c|}{ Std. Err. } & \multicolumn{1}{c|}{$\mathrm{t}$} & \multicolumn{2}{c|}{$\mathrm{P}>\mathrm{It} \mid$} & \multicolumn{2}{|c|}{ [95\% Conf. Interval] } \\
\hline In_part & 0.279 & 0.201 & 1.390 & 0.167 & -0.117 & 0.675 \\
\hline In_aport & -0.664 & 0.215 & -3.090 & 0.002 & -1.089 & -0.240 \\
\hline In_itaee & 0.634 & 0.270 & 2.350 & 0.020 & 0.102 & 1.166 \\
\hline _cons & -8.167 & 1.185 & -6.890 & 0.000 & -10.501 & -5.834 \\
\hline
\end{tabular}

\begin{tabular}{|c|c|c|c|c|}
\hline sigma_u & 0.665 & & & \\
\hline sigma_e & 0.217 & & & \\
\hline rho & \multicolumn{4}{|c|}{0.903 (fraction of variance due to $u \_i$ ) } \\
\hline F test that all $u_{-} i=0$ : & $F(30,245)=$ & 75.97 & Prob $>F=$ & 0.000 \\
\hline
\end{tabular}


210 ECONOMÍA TEORÍA Y PRÁCTICA • Nueva Época, número 47, julio-diciembre 2017

\begin{tabular}{|c|c|c|c|c|}
\hline \multicolumn{3}{|c|}{ Random-effects GLS regression } & \multirow{2}{*}{$\begin{array}{c}\text { Number of obs }= \\
\text { Number of groups= }\end{array}$} & \multirow{2}{*}{$\begin{array}{l}279 \\
9\end{array}$} \\
\hline Group variable: & entidad & & & \\
\hline \multirow[t]{2}{*}{ R-sq: } & within $=$ & 0.000 & Obs per group: & $\min =9$ \\
\hline & between= & 0.000 & $\operatorname{avg}=$ & 9.0 \\
\hline $\max =22$ & overall= & 0.000 & $\max =$ & 9 \\
\hline
\end{tabular}

\begin{tabular}{|l|c|c|c|c|}
\hline Ingastopublicopercapita & Coef. & Std. Err. & $t$ & $P>|t|$ \\
\hline \multicolumn{5}{|l|}{} \\
\hline In_part & 0.018 & 0.016 & 1.157 & 0.248 \\
\hline In_aport & 0.013 & 0.011 & 1.181 & 0.239 \\
\hline In_itaee & -0.042 & 0.004 & -11.850 & 0.000 \\
\hline _cons & 0.000 & 0.000 & 0.771 & 0.442 \\
\hline
\end{tabular}

\begin{tabular}{|r|l|r|r|l|}
\hline Media de la vble. Dep. & 0.010 & \multirow{5}{*}{} & D.T. de la vble. dep. & 0.013 \\
\hline Suma de cuad. residuos & 0.049 & D.T. de la regresión & 0.013 \\
\hline Log-verosimilitud & 811.486 & & Criterio de Akaike & -1614.971 \\
\hline Criterio de Schwarz & -1600.446 & & Crit. de Hannan-Quinn & -1609.144 \\
\hline
\end{tabular}




\section{Anexo 4. Prueba Haussman}

\begin{tabular}{|c|c|c|c|c|}
\hline \multicolumn{5}{|c|}{. hausman efectos_fijos efectos_aleatorios } \\
\hline \multicolumn{5}{|c|}{ Coefficients } \\
\hline & (b) & (B) & $(b-B)$ & $\operatorname{sqrt}\left(\operatorname{diag}\left(\mathrm{V} \_b-V \_B\right)\right)$ \\
\hline & efectos_fijos & efectos_aleatorios & Difference & S.E. \\
\hline In_part & 0.279 & 0.160 & 0.119 & 0.079 \\
\hline In_aport & -0.664 & -0.514 & -0.150 & 0.091 \\
\hline In_itaee & 0.634 & 0.763 & -0.129 & 0.078 \\
\hline
\end{tabular}

$\mathrm{b}=$ consistent under $\mathrm{Ho}$ and $\mathrm{Ha}$; obtained from xtreg

$\mathrm{B}=$ inconsistent under $\mathrm{Ha}$, efficient under $\mathrm{Ho}$; obtained from xtreg

Test: Ho: difference in coefficients not systematic

$\operatorname{chi} 2(3)=(b-B)^{\prime}\left[\left(V \_b-V \_B\right)^{\wedge}(-1)\right](b-B)$

$=4.56$

Prob $>$ chi $2=0.207$

( $V \_b-V_{-} B$ is not positive definite) 
212 ECONOMÍA TeOrÍA Y PrÁCTICA • Nueva Época, número 47, julio-diciembre 2017

\section{Anexo 5. Prueba Wooldrige}

\begin{tabular}{|c|r|l|}
\hline .xtserial In_esf_fis In_part In_aport In_itaee, output \\
\hline Linear regression & Number of obs $=$ & 248 \\
\hline & Prob $>$ F $=$ & 0.017 \\
\hline & R-squared $=$ & 0.033 \\
\hline \multicolumn{2}{|l|}{ Root MSE $=$} & 0.247 \\
\hline (Std. Err. adjusted for 31 clusters in entidad) & & \\
\hline
\end{tabular}

\begin{tabular}{|l|c|c|c|c|c|c|}
\hline D. In_esf_fis & Coef. & Std. Err & $\mathrm{t}$ & \multicolumn{2}{|c|}{ P>I t I } & \multicolumn{2}{|c|}{ [95\% Conf. Interval] } \\
\hline In_part & & & & & & \\
\hline D1. & -0.016 & 0.141 & -0.120 & 0.909 & -0.304 & 0.271 \\
\hline In_aport & & & & & & \\
\hline D1. & -0.390 & 0.190 & -2.050 & 0.049 & -0.779 & -0.001 \\
\hline In_itaee & & & & & & \\
\hline D1. & 0.277 & 0.361 & 0.770 & 0.449 & -0.459 & 1.013 \\
\hline
\end{tabular}

\begin{tabular}{|l|l|}
\hline Wooldridge test for autocorrelation in panel data \\
\begin{tabular}{|l|l|}
\hline HO: no first-order autocorrelation \\
\hline Prob $>F=$ & 0.001 \\
\hline
\end{tabular}
\end{tabular}




\section{Anexo 6. Prueba Breush-Pagan}

\begin{tabular}{|l|}
\hline .quietly xtreg In_esf_fis In_part In_aport In_itaee, re \\
\hline .xttest0 \\
\hline Breush and Pagan Lagrangian multiplier test for random effects \\
\hline In_esf_fis[entidad,t] $=\mathrm{Xb}+\mathrm{u}[$ entidad] + e[entidad,t] \\
\hline
\end{tabular}

\begin{tabular}{|c|c|c|}
\hline \multicolumn{2}{|l|}{ Estimated results: } & \\
\hline & Var & sd= sqrt (Var) \\
\hline In_esf_-s & 0.436 & 0.660 \\
\hline $\mathrm{e}$ & 0.047 & 0.217 \\
\hline $\mathrm{u}$ & 0.402 & 0.634 \\
\hline
\end{tabular}

\begin{tabular}{|r|l|}
\hline Test: $\operatorname{Var}(\mathrm{u})=$ & 0 \\
\hline chibar2 $(01)=$ & 858.55 \\
\hline Prob $>$ chibar2 $=$ & 0.000 \\
\hline
\end{tabular}


214 ECONOMÍA TEORÍA Y PRÁCTICA • Nueva Época, número 47, julio-diciembre 2017

\section{Anexo 7. Estimación Mínimos Cuadrados Generalizados Factibles}

\begin{tabular}{|l|}
\hline .xtgls In_esf_fis In_part In_aport In_itaee, panels (hetero) \\
\hline Cross-sectional time-series FGLS regression \\
\hline
\end{tabular}

\begin{tabular}{|r|l|}
\hline Coefficients: & generalized least squares \\
\hline Panels: & heteroskedastic \\
\hline Correlation: & no autocorrelation \\
\hline
\end{tabular}

\begin{tabular}{|r|l|l|r|l|}
\hline Estimated covariances $=$ & 31 & & Number of obs $=$ & 279 \\
\hline Estimated autocorrelations $=$ & 0 & & Number of groups $=$ & 31 \\
\hline Estimated coefficients $=$ & 4 & & Time periods $=$ & 9 \\
\hline & & & Wald chi2 $(3)=$ & 43.21 \\
\hline & & & Prob $>$ chi2 $=$ & 0.000 \\
\hline
\end{tabular}

\begin{tabular}{|l|c|c|c|c|c|c|}
\hline In_esf_fis & Coef. & Std. Err. & $z$ & $P>|z|$ & \multicolumn{2}{|c|}{ (95\% Conf. Interval) } \\
\hline In_part & -0.261 & 0.082 & -3.190 & 0.001 & -0.421 & -0.101 \\
\hline In_aport & -0.177 & 0.079 & -2.240 & 0.025 & -0.332 & -0.022 \\
\hline In_itaee & 0.798 & 0.218 & 3.660 & 0.000 & 0.371 & 1.225 \\
\hline _cons & -9.056 & 0.953 & -9.50 & 0.000 & -10.925 & -7.187 \\
\hline
\end{tabular}




\section{Anexo 8. Prueba Ramsey- Reset}

\begin{tabular}{|l|}
\hline .xtgls In_esf_fis In_part In_aport In_itaee yhat2, panels(hetero) \\
\hline Cross-sectional time-series FGLS regression \\
\hline Coefficients: generalized least squares \\
\hline Panels: heteroskedastic \\
\hline Correlation: no autocorrelation \\
\hline
\end{tabular}

\begin{tabular}{|c|c|c|c|}
\hline Estimated covariences= & 31 & Number of obs= & 279 \\
\hline Estimated autocorrelations= & 0 & Number of groups= & 31 \\
\hline Estimated coefficients $=$ & 5 & Time periods $=$ & 9 \\
\hline & & Wald chi2 $(3)=$ & 40.68 \\
\hline & & Prob $>$ chi $2=$ & 0.000 \\
\hline
\end{tabular}

\begin{tabular}{|l|c|c|c|c|c|c|}
\hline In_esf_fis & Coef. & Std. Err. & $z$ & P>|z| & \multicolumn{2}{|c|}{ (95\% Conf. Interval) } \\
\hline In_part & 0.141 & 3.253 & 0.040 & 0.965 & -6.235 & 6.516 \\
\hline In_aport & 0.120 & 2.196 & 0.050 & 0.957 & -4.185 & 4.424 \\
\hline In_itaee & -0.527 & 10.073 & -0.050 & 0.958 & -20.270 & 19.215 \\
\hline yhat2 & -0.170 & 1.273 & -0.130 & 0.894 & -2.665 & 2.325 \\
\hline _cons & 1.890 & 82.953 & 0.020 & 0.982 & -160.695 & 164.475 \\
\hline
\end{tabular}


